\title{
Progress Review on Topological Properties of Heusler Materials
}

\author{
Zhi $\operatorname{Lin}^{1, *}$ \\ ${ }^{1}$ Institute for Advanced Study Shenzhen University Shenzhen, China
}

\begin{abstract}
Starting from crystal, electronic and magnetic structures of Heusler compounds, this paper studies the new topological materials related to Heusler compounds and their topological properties, such as anomalous Hall effect, skyrmions, chiral anomaly, Dirac fermion, Weyl fermion, transverse Nernst thermoelectric effect, thermal spintronics and topological surface states. It can be discovered that the topological state of Heusler compound can be well protected due to its high symmetry, thus producing rich topological properties. Heusler materials belonged to Weyl semimetals usually have strong anomalous Hall effect, and the Heusler materials with doping or Anomalous Nernst Effect (ANE) usually have higher thermoelectric figure of merit. These anomalous effects are closely related to the strong spin-orbit interaction. In application, people can use the non-dissipative edge state of quantum anomalous Hall effect to develop a new generation of low-energy transistors and electronic devices. The conversion efficiency of thermoelectric materials can be improved by ANE, and topological superconductivity can be used to promote the progress of quantum computation.
\end{abstract}

\section{INTRODUCTION}

With Moore's law coming to an end, the upgrading of chips makes demands of the innovation of theory and materials. The topological insulator has great potential. Such material is insulated in the interior while the interface allows the charge to move, so there is less heat consumption inside topological insulator. At the same time, the topological insulator only has a thin conducting layer on the surface, which means it is easy to manipulate the electron spin through the surface states, and it can also be used in quantum computation. Heusler compound is one of the materials that can be used in topological insulator. It has many physical properties, such as anomalous Hall effect, anomalous Nernst effect, thermal spintronics and so on. Also, the relation the Weyl fermion needs is satisfied in Heusler compound naturally, that is, the inversion symmetry is broken naturally. Therefore, the Heusler compound and Weyl semimetals can be studied simultaneously.

The general formula of Heusler material has already been known, and nowadays, experimental physicists are trying to combine different elements. However, there are no general theories to predict a more practical Heusler material or Weyl semimetal. At present, the experimental results are too scattered, and their theoretical clues are not clear. This paper attempts to study the topological properties of Heusler compound from its basic structure, so as to discover and summarize the rules.

\section{Heusler Materials}

\subsection{Introduction}

The Heusler compound was first discovered in 1903. While studying $\mathrm{Cu} 2 \mathrm{MnAl}$ at room temperature, Fritz Heusler found that although all elements in $\mathrm{Cu} 2 \mathrm{MnAl}$ were nonferromagnetic elements, the whole compound was ferromagnetic. In the follow-up study, it was found that Heusler materials also have other unique physical properties, such as high controllability. Under different conditions, Heusler materials can have the properties of semiconductor, metal, superconductor or semimetal respectively. In addition, various magnetic behaviors and multi-functional magnetism of Heusler materials have attracted much attention [1].

In terms of the components, Heusler materials are intermetallic compounds, which can be divided into two categories of Full-Heusler $(\mathrm{FH})$ compounds and HalfHeusler $(\mathrm{HH})$ compounds. Among them, FH compounds have the general formula of X2YZ, which can be further subdivided into Regular-Heusler and Inverse-Heusler compounds. In Regular-Heusler compounds, X is usually a transition metal or other elements with more electropositivity. $\mathrm{Y}$ is a transition metal, alkaline-earth metal or rare earth element. And $\mathrm{Z}$ is the main group element. In Inverse-Heusler compounds, which is relatively rare, one of the two $\mathrm{X}$ atoms will exchange positions with the $\mathrm{Y}$ element [1],[2]. For HH compound, its chemical composition is XYZ. 


\subsection{Crystal Structure}

The bonding between atoms directly affects the structural characteristics of Heusler compounds. Only the atoms that with small electronegativity difference can form the sublattice of Heusler materials easily. Specifically, Regular-Heusler is composed of four interpenetrating face centered cubic Bravais lattices with L21 structure and No.225 (Fm $\overline{3} \mathrm{~m})$ space group. It is nonpolar due to its central symmetry. The Inverse-Heusler and Half-Heusler are composed of three interpenetrating face centered cubic Bravais lattices. Their space group is No.216 (F $\overline{4} 3 \mathrm{~m}$ ), which is non-centrosymmetric, so it contributes to polarity. On the whole, the symmetry of the crystal structure of Heusler material is stronger than that of most other materials.

In Heusler compounds, the different atomic occupation of the crystal will affect the band structure of the material, and then affect the topological properties and application functions. Moreover, under the conditions of different material preparation methods and different heat treatment processes, even the materials with same composition can have different crystal structure and magnetic properties, and the electronic structure, magnetism and phase transition will change accordingly, which will ultimately affect the topological physical properties and functions of the materials.

\subsection{Electronic Structure}

Heusler compound can work as conductor, insulator, topological insulator, semiconductor or semimetal. The key factors that matter are its band and band gap. In conductor, the conduction band and the valence band will overlap, so the electrons can move freely in the band. In insulator, the gap between conduction band and valence band is huge. The gap also exists in topological insulator. However, at the same time, the topological surface state allows electron conduction. In semiconductor, the band gap is smaller than that of insulator, so the electron can jump from the valence band to the conduction band at higher temperature. If the semiconductor is intrinsic, the Fermi level will lie exactly in the middle of the forbidden band. In semimetals, the bottom of conduction band and the top of valence band just meet at a point, as shown in Fig. 1, so it can conduct electricity even if it is at very low temperature.

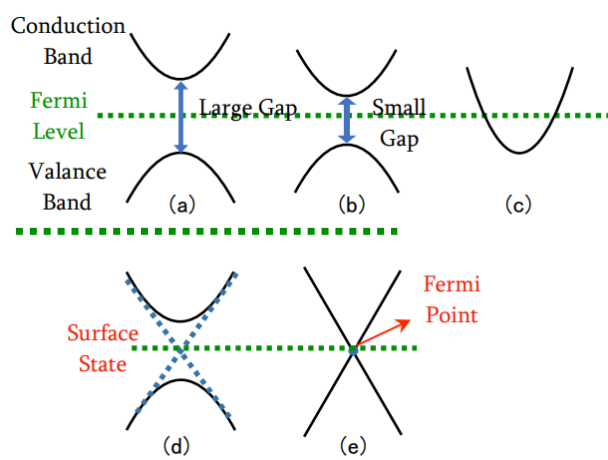

Figure 1. Band structure of different materials: (a) insulator; (b) semiconductor; (c) conductor; (d) topological insulator; (e) topological semimetal.
The electronic structures of $\mathrm{FH}$ and $\mathrm{HH}$ are quite different. For FH compounds, they usually have HgTe-like band structures. If FH wants to be a semiconductor, the total number of valence electrons should be 24 , so that the outermost electron layer can reach a full shell state, such as Fe2VAl. When the total number of valence electrons is not 24, there will appear ferromagnetism, ferrimagnetism or antiferromagnetism.

For $\mathrm{HH}$ compound, if the total number of valence electrons is 18 , it will be a semiconductor material. In addition, some materials with 8 valence electrons are also Heusler semiconductors with Nowotny-Juza phase, such as LiAlSi, which usually occurs in the presence of light metals When the total number of valence electrons in HF compounds is not 18 , the compounds will tend to become metal or magnetic materials. At this time, most of them do not crystallize in the configuration of $\mathrm{Clb}$, such as $\mathrm{FeMnSb}$ $\mathrm{CrMnSb}, \mathrm{MnCoSb}$ [3]. The reason is that they contain more than one magnetic element, and the electrons of $\mathrm{Mn}$ ions are not completely bound in the ions, which lead to instability [3]. There are also exceptions, such as $\mathrm{NiMnSb}$ and PtMnSb, which are known as semimetallic ferromagnets, will crystallize in the configuration of $\mathrm{C} 1 \mathrm{~b}$. According to different spin directions, these semimetals can exhibit both metal and insulator properties in the same material.

Different electronic structures may affect the conductivity and thermoelectricity of Heusler material. For thermoelectricity, some $\mathrm{HH}$ materials can be further analyzed. As shown in Fig. 2 [4],[5], it can be seen from the band structure that the peak of valence band of $\mathrm{HfCoSb}$ $\mathrm{ZrCoSb}$ [4]、 $\mathrm{ZrNiSn}$ [5] is at L point, and the bottom is at $\mathrm{X}$ point. Among these compounds, the Co-d state and $\mathrm{Zr}(\mathrm{HF})-\mathrm{d}$ state, Ni-d state and $\mathrm{Zr}$-d state are highly hybrid. The chighest points of valence band in $\mathrm{ZrIrSb}$ and $\mathrm{HfIrSb}$ compounds are at $\Gamma$ point, and the hybridization of the $\mathrm{Zr}(\mathrm{HF})-\mathrm{d}$ state and the antibonding low energy state produced by iridium's s-orbital leads to the lowest point in conduction band at $\Gamma$ point [4]. These different configurations of the conduction band further determine their thermoelectric properties (see "2.2 thermoelectricity" for details). (a) $\mathrm{HfCoSb}$

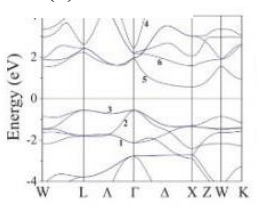

(b) $\mathrm{ZrCoSb}$

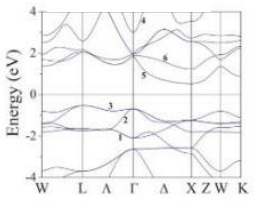

(c) $\mathrm{ZrNiSn}$

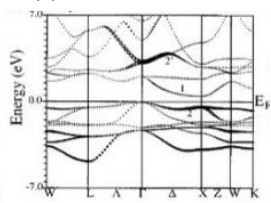

Figure 2. The band structure of HH (a) HfCoSb [4], (b) $\mathrm{ZrCoSb}$ [4], (c) ZrNiSn [5] near Fermi level along W-L- $Г-X-$ $\mathrm{W}-\mathrm{K}$ direction (set Fermi energy as zero)

As for the conductivity, it is known that HH LaPtBi [6] and $\mathrm{NiMnSb}$ [7], $\mathrm{FH} \mathrm{Cr} 2 \mathrm{MnZ}(\mathrm{Z}=\mathrm{P}, \mathrm{As}, \mathrm{Sb}, \mathrm{Bi}$ ) (this series of protocells contain 24 valence electrons) [8] and Fe2MnSi [9] are semimetals at a certain temperature. Through calculation and prediction, it can also be known that $\mathrm{NiSnM}(\mathrm{M}=\mathrm{Ti}, \mathrm{Zr}, \mathrm{Hf})$ is a semiconductor with small energy gap, and $\mathrm{Ni} 2 \mathrm{SnM}(\mathrm{M}=\mathrm{Ti}, \mathrm{Zr}, \mathrm{Hf})$ is a normal metal [10]. 
To be more specific, for $\mathrm{LaPtBi}$, the band structure is semimetal (as shown in Fig. 3). In terms of crystal structure, its symmetry is high (Fig. 4), so the topological phase is well protected. Therefore, LaPtBi can be a topological semimetal.

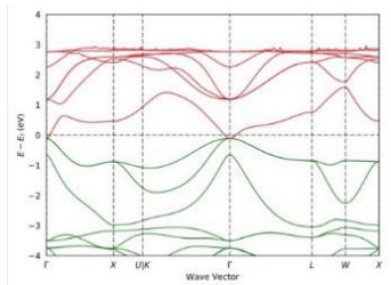

Figure 3. LaPtBi Non-SOC band structure (http://materiae.iphy.ac.cn/search)

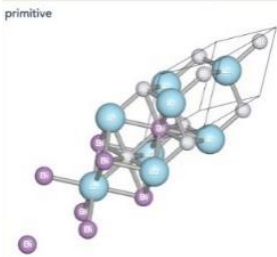

Figure 4. LaPtBi crystal structure (http://materiae.iphy.ac.cn/search)

It can be seen that materials with different conductivity, magnetism and thermoelectric properties can be obtained by using elements with different valence electron numbers to form Heusler compounds or by a series of external control. The high modulation and wide application prospects of Heusler materials can be seen as well.

\subsection{Magnetic Structure}

In addition to the charge, electrons also have an intrinsic property of spin. The spin magnetic moment produced by the electron spin and the orbital magnetic moment produced by electrons orbiting the nucleus make the material magnetic. There is a certain relationship between the valence electron concentration and magnetic moment of the Heusler material, which can be analyzed by the Slater-Pauling curve. The total spin magnetic moments of some Heusler materials can also be obtained according to the Slater-Pauling rule. According to Slater Pauling rule [11], we can get

$$
m=N_{v}-24
$$

Here, $m$ is the magnetic moment per unit chemical formula, deduced from the electron difference between the upper and lower spins, and $\mathrm{Nv}$ is the total number of electrons. It can also be seen from the experiment that for Co2-based compounds with semimetallic ferromagnetism, the magnetic moment and valence electron number present a linear relationship, but there is no obvious relationship between the magnetic moment and the valence electron number of many Fe2-based and Ni2-based compounds [1]. In addition, because the $\mathrm{X}$ atom in $\mathrm{FH}$ occupies two tetrahedral sites and can interact in the sublattice, there are two magneton lattices and have more abundant magnetic properties.

In $\mathrm{HH}$ compounds, a similar equation is

$$
\mathrm{m}=\mathrm{N}_{\mathrm{v}}-18
$$

In $\mathrm{HH}$, only the atoms on octahedral site have magnetic moment, so there is only one magneton lattice. In addition, if the number of electrons is more than 18 , the system is magnetically stable.

The magnetic properties of Heusler compound and their

\begin{tabular}{|c|c|c|c|}
\hline TABLE I. & MAGNETIC & ROPERTIES OF H & SLER COMPO \\
\hline Properties & Discoverer & $\begin{array}{c}\text { Representative } \\
\text { Materials }\end{array}$ & Statement \\
\hline $\begin{array}{l}\text { Ferromag- } \\
\text { netic }\end{array}$ & $\begin{array}{c}\text { F.Heusler } \\
\text { (1903) }\end{array}$ & $\mathrm{Cu} 2 \mathrm{MnAl}$ & $\begin{array}{c}\mathrm{Cu} \backslash \mathrm{Mn} \backslash \mathrm{Al} \text { are } \\
\text { nonferromagnetic } \\
\text { elements. }\end{array}$ \\
\hline $\begin{array}{l}\text { Antiferro- } \\
\text { magnet [12] }\end{array}$ & $\begin{array}{l}\text { DP Oxley } \\
(1962)\end{array}$ & $\mathrm{Cu} 2 \mathrm{MnSb}$ & $\begin{array}{c}\text { This material can } \\
\text { also be } \\
\text { ferromagnetic. }\end{array}$ \\
\hline $\begin{array}{c}\text { Crystal } \\
\text { structure } \\
\text { containing } \\
\text { rare earth } \\
\text { element [13] }\end{array}$ & $\begin{array}{l}\text { R.Marazza et } \\
\text { al. (1980) }\end{array}$ & $\mathrm{RPdSb} / \mathrm{RPdBi}$ & $\begin{array}{c}\mathrm{R}=\text { rare earth } \\
\text { element }\end{array}$ \\
\hline $\begin{array}{l}\text { Half-metallic } \\
\text { ferromag- } \\
\text { netism [1] }\end{array}$ & $\begin{array}{l}\text { De Groot et } \\
\text { al. / Kübler } \\
\text { et al. (1983) }\end{array}$ & $\mathrm{MnNiSb} / \mathrm{Co} 2 \mathrm{MnSn}$ & $/$ \\
\hline $\begin{array}{c}\text { Antiferroma } \\
\text { gnet at low } \\
\text { temperature } \\
{[14]}\end{array}$ & $\begin{array}{l}\text { S. K. Malik } \\
\text { et al. (1985) }\end{array}$ & $\mathrm{Pd} 2 \mathrm{RSn}(\mathrm{R}=\mathrm{Tb}-\mathrm{Yb})$ & $\begin{array}{l}\mathrm{Tb}-\mathrm{Yb} \text { are rare } \\
\text { earth element. }\end{array}$ \\
\hline $\begin{array}{c}\text { Antiferroma } \\
\text { gnet at low } \\
\text { temperature } \\
{[15]}\end{array}$ & $\begin{array}{l}\text { G.André et } \\
\text { al. (1997) }\end{array}$ & HoPdSb & $\begin{array}{l}\text { Ho is rare earth } \\
\text { element. }\end{array}$ \\
\hline $\begin{array}{l}\text { Paramagneti } \\
\mathrm{c} \text { at low } \\
\text { temperature } \\
{[16]}\end{array}$ & $\begin{array}{l}\text { Kaczorowski } \\
\text { D. et al. } \\
\text { (2005) }\end{array}$ & $\operatorname{ErPdA}(\mathrm{A}=\mathrm{Sb} / \mathrm{Bi})$ & $\begin{array}{l}\text { Er is rare earth } \\
\text { element. }\end{array}$ \\
\hline $\begin{array}{l}\text { Spin Gapless } \\
\text { Semicon- } \\
\text { ductors) [17] }\end{array}$ & $\begin{array}{l}\text { Ouardi, S. Et } \\
\text { al. (2013) }\end{array}$ & $\mathrm{Mn} 2 \mathrm{CoAl}$ & / \\
\hline
\end{tabular}
discoveries can be seen in Table 1 .

\section{TOPOLOGICAL PROPERTIES OF HEUSLER MATERIALS}

\subsection{Weyl Semimetals, Topological States, and Anomalous Hall Effect}

Since 2006, it has been found that the fully-occupied electron energy band has topological properties. With topology involved in, metals can also be divided into ordinary metals and topological semi metals. These topological materials have great application value in the field of optics and quantum information.

In $1929, \mathrm{H}$. Weyl pointed out that the massless solution of the Dirac equation described a pair of new particles with opposite chirality and half integer spin, which are then called Weyl fermions. Weyl fermions are low-energy excitations of electrons, which usually occur at the intersection of two nondegenerate electron energy bands in the lattice momentum space. These intersections are called Weyl points, which are magnetic monopoles of gauge field defined by Berry curvature. Their magnetic charge is the quantum number of the magnetic flux passing through the Fermi surface which covers the intersection of the energy levels [18]. Further researches then discovered Weyl semimetal (WSM), a real material which is only composed of Weyl fermions or band intersections. The density of states on the Fermi surface of WSM is zero, and WSM can show the properties of metal and insulator at the same time. This is due to the fact that the Weyl points in solids always appear in pairs. Under the equilibrium state, the current 
contribution of Weyl point with different chirality is strictly offset, and the macro-current of the system is zero. However, once a nonequilibrium state is formed by an external field, which then results in an unequal chemical potential at the Weyl points with different chirality, the chiral magnetic effect will lead to a chiral current that is determined by the direction of magnetic field and the chirality of the Weyl point but any crystal direction or other material details. Only surface electrons moving at a specific speed and direction can enter the interior of the crystal.

WSM is a kind of topological semimetallic materials. According to the degeneracy and shape of band crossing points, topological semimetals also include Dirac semimetals with four-fold degenerate band-touching points and nodal-line semimetals with two-fold degenerate bandtouching points. However, through specific symmetry breaking, these two topological semimetallic states protected by symmetry can be transformed into Weyl semimetallic states. For example, the Dirac semimetal is transformed into WSM under time reversal symmetry breaking, and when the mirror symmetry is broken, the nodal-line semimetal is transformed into a Weyl semimetal [19].

WSM is divided into Type I and Type II. Type I WSM corresponds to Lorentz symmetry, while Type II WSM does not. The Weyl cone of II-WSM shows a strong tilt, so that the Weyl point appears as the contact point of the electron band and the hole band on the Fermi surface (as shown in Fig. 5 [20], [21]). The hourglass shape in the picture is the Weyl cone. Weyl points with different chiral appear at different momentum positions, and their projections on the surface are connected by the Fermi arc.
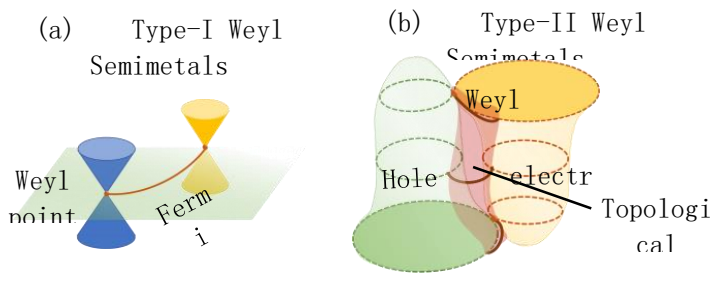

Figure 5. The electronic structure of two types of WSM (a)Type I and (b)Type II

Because the emergence of WSM needs to break the time reversal symmetry or space inversion symmetry, and in $\mathrm{IH}$ and $\mathrm{FH}$, inversion symmetry is naturally broken, so many Heusler compounds are Weyl semimetals, such as LnPtBi ( $\mathrm{Ln}=\mathrm{Y}, \mathrm{Gd}$, or Nd) [22], WTeS and WTeSe [23], LaAlGe [24], and Co2TiX (X=Si, Ge, or Sn) [25] in FH. The time reversal symmetry of magnetic WSM is guaranteed by the system magnetism and can be controlled by the external field. It is easy to control the topological state by temperature or magnetic field, so it has great application potential. WSM has been found to have many important topological properties, such as chiral anomaly [26],[27] and the negative magnetoresistance induced by it [28], Fermi arc surface state and Dirac cone bulk state [29], etc. What to be illustrated in detail is the strong giant anomalous Hall effect produced and regulated by the topology related Berry curvature.

Hall effect is generally considered as an external mechanism: the positive and negative charges of motion are respectively gathered on two boundaries of the sample under the action of Lorentz force. However, the anomalous Hall effect is produced by the spontaneous polarization of the material itself without the involvement of external magnetic field. Therefore, the Hall effect taking place in zero field is anomalous Hall effect. Magnetic Weyl semimetals and topological insulators in Heusler compound usually have very strong quantum anomalous Hall effect. Their common characteristic is that they both have topological surface states, which satisfy the conditions of anomalous Hall effect.

In 2013, Professor Xue Qikun and his team observed quantum anomalous Hall effect in magnetic topological insulator for the first time [20]. In this case, it is the edge state that contributes to the quantum conductivity, that is, the conductive electrons move on the material boundary. If the strong spin orbit coupling is embedded in the magnetic material, the topological Hall effect will be produced. In Weyl semimetals, the intrinsic anomalous Hall effect is even greater, because the cross-section line and Weyl point of the spin coupling open energy system will produce strong Berry curvature, which has a significant lateral effect on the moving carriers

In the inverse Heusler alloys (magnetic semimetal) like $\mathrm{Mn} 2 \mathrm{YZ}$, where $\mathrm{Y}$ is the transition metal and $\mathrm{Z}$ is the dominant group element, there is often competition between ferromagnetic and antiferromagnetic interactions. In general, these compounds are not centrosymmetric chiral magnets. Therefore, the existence of DzyaloshinskiiMoriya interaction and the magnetic crystal anisotropy caused by tetragonal distortion lead to noncollinear spin orientation and stable skyrmion [30]. When solving the nonlinear field theory equation, a kind of "particle" protected by topology will be obtained. Its twodimensional spin structure can be continuously projected onto a sphere, inside which an "equivalent" magnetic monopole can be considered to exist. The magnetic skyrmion can produce an equivalent magnetic field for the electrons flowing through its own topological spin structure. This magnetic field comes from the interior of the material, so it can produce anomalous Hall effect. Similarly, the Weyl point (intersection of two nondegenerate states) in the momentum space of Weyl semimetals, such as RPtBi ( $\mathrm{R}=\mathrm{Gd}$ or $\mathrm{Nd})$ [31] can also be regarded as Berry curvature's magnetic monopole, resulting in anomalous Hall effect. (A further introduction of skyrmion will be placed in the "2.3 thermal spintronics".)

For ferromagnetic WSM, the extrinsic AHE disappears completely, leaving the intrinsic AHE only, and the intrinsic part is all determined by the relative position and topological charges of Weyl nodes. When the Chern number of each Fermi surface is non-zero and the Fermi level is close to Weyl node, the performance of AHE is almost completely independent of the properties of the Fermi surface. This is quite different from the general ferromagnetic metals. For general ones, even if there is anomalous Hall effect, it will still include the contribution of the Fermi surface and the extrinsic AHE [32]. 


\subsection{Thermoelectricity and Topologically Enhanced Transverse Nernst Thermoelectric Effect}

Thermoelectric effect is a phenomenon that temperature difference and voltage, or electric energy and heat energy transform into each other, including Seebeck effect, Peltier effect and Thomson effect. Among them, the Seebeck effect is the most basic form of thermoelectric effect, and it is also the most common one in HF compound. The Seebeck effect is produced by the accumulation of charges or currents generated by temperature difference. Under the temperature gradient, the carriers move from the hot end to the cold end and accumulate at the latter, thus forming a potential difference inside the material, and generating a reverse charge flow caused by the potential difference. When charges doing thermal motion reach a dynamic balance with the internal electric field, a stable thermoelectric force will be formed between two ends of the materials, which can be measured by Seebeck coefficient.

The material with thermoelectric effect is thermoelectric material. Good thermoelectric materials are generally semiconductor materials with narrow band gap, high carrier mobility and low thermal conductivity. The thermoelectric efficiency can be got from the equation

$$
\mathrm{ZT}=\frac{\mathrm{S}^{2} \sigma \mathrm{T}}{\kappa}
$$

Here, $\mathrm{S}$ is the Seebeck coefficient, $\sigma$ is conductivity, $T$ is absolute temperature, and $\kappa$ is thermal conductivity, including the contribution from electrons $\kappa_{\mathrm{e}}$ and lattice vibration $\kappa_{\mathrm{p}}$. HH have the potential to become good thermoelectric materials, especially those with 18 -electron structure in the outermost layer, such as MNiSn, $\mathrm{MCoSb}$ $(\mathrm{M}=\mathrm{Ti}, \mathrm{Zr}, \mathrm{Hf}), \operatorname{ArIrX}(\mathrm{X}=\mathrm{As}, \mathrm{Sb}, \mathrm{Bi})$ and other compounds [17].

At room temperature $(300 \mathrm{k})$ and without doping, according to the $S, \sigma$ and $\kappa$ parameters in relevant literatures, the thermoelectric merit value ZT (see Table 2) can be calculated by formula (3) and plotted in Fig. 6 $[5],[33],[34]$. It can be seen from the figure that the thermoelectric performance of ErNiSb is very strong, while that of $\mathrm{TiCoSb}$ is poor. This may be because the average relative atomic mass of the former is larger. The lower the lattice vibration frequency, the lower the lattice thermal conductivity and the higher the thermoelectric merit value. TABLE II. THE THERMOELECTRIC PROPERTIES OF DIFFERENT

\begin{tabular}{|c|c|c|c|c|}
\hline \multirow{2}{*}{ Hempound } & $\boldsymbol{\rho}(\mathbf{\Omega c m})$ & $\boldsymbol{\kappa}(\mathbf{W} /(\mathbf{m} \cdot \mathbf{K}))$ & $\mathbf{S}(\boldsymbol{\mu} \mathbf{V} / \mathbf{K})$ & $\mathbf{Z T}(\mathbf{i n} \mathbf{S I})$ \\
& & & & \\
\hline ZrNiSn [33] & $1.10 \times 10^{-2}$ & 8.8 & -176 & 0.10 \\
\hline HfNiSn [33] & $1.30 \times 10^{-2}$ & 6.7 & -124 & 0.05 \\
\hline TiNiSn [33] & $1.00 \times 10^{-2}$ & 9.3 & -142 & 0.07 \\
\hline TiCoSb [34] & $4.00 \times 10^{-2}$ & 20 & -250 & 0.02 \\
\hline TmNiSb [5] & $3.00 \times 10^{-3}$ & 2.8 & 60 & 0.13 \\
\hline ErNiSb [5] & $3.00 \times 10^{-3}$ & 4 & 160 & 0.64 \\
\hline
\end{tabular}

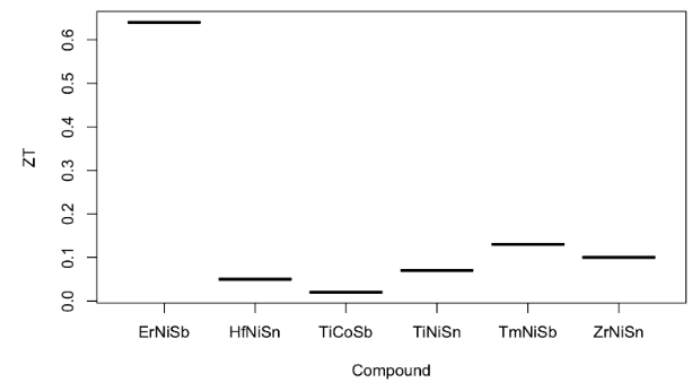

Figure 6 . Comparison of thermoelectricity of different Heusler compound $(300 \mathrm{~K})$

In order to improve the merit value of thermoelectricity further, there are two methods. One is to dope the material. The other is to use anomalous Nernst effect. For doping, $\mathrm{HH}$ materials are used more often. As shown in Table 3 and Fig. 7, HH with reasonable doping has higher $\mathrm{ZT}$ value. TABLE III. MAXIMUM MERIT VALUE OF THERMOELECTRICITY OF DOPED HH (DIFFERENT TEMPERATURE FOR DIFFERENT COMPOUNDS ) [35]-[44]

\begin{tabular}{|c|c|r|}
\hline & Compound & $\mathrm{ZT}_{\max }$ \\
\hline $\mathrm{a}[35]$ & $\mathrm{Hf}_{0.75} \mathrm{Zr}_{0.25} \mathrm{NiSn}_{0.975} \mathrm{Sb}_{0.025}$ & 0.8 \\
\hline $\mathrm{b}[36]$ & $\mathrm{Ti}_{0.95} \mathrm{Hf}_{0.05} \mathrm{NiSn}_{0.99} \mathrm{Sb}_{0.01}$ & 0.78 \\
\hline $\mathrm{c}[37]$ & $\mathrm{Zr}_{0.5} \mathrm{Hf}_{0.5} \mathrm{Ni}_{0.8} \mathrm{Pd}_{0.2} \mathrm{Sn}_{0.99} \mathrm{Sb}_{0.01}$ & 0.7 \\
\hline $\mathrm{d}[38]$ & $\mathrm{Zr}_{0.3} \mathrm{Hf}_{0.65} \mathrm{Ta}_{0.05} \mathrm{NiSn}$ & 0.85 \\
\hline $\mathrm{e}[39]$ & $\mathrm{Ti}_{0.3} \mathrm{Zr}_{0.35} \mathrm{Hf}_{0.35} \mathrm{NiSn}$ & 0.82 \\
\hline $\mathrm{f}[40]$ & $\mathrm{Ti}_{0.37} \mathrm{Zr}_{0.37} \mathrm{Hf}_{0.26} \mathrm{NiSn}$ & 1 \\
\hline $\mathrm{g}[41]$ & $\mathrm{Ti}_{0.5} \mathrm{Zr}_{0.25} \mathrm{Hf}_{0.25} \mathrm{NiSn}_{0.998} \mathrm{Sb}_{0.002}$ & 1.2 \\
\hline $\mathrm{i}[43]$ & $\mathrm{Ti}_{0.5}\left(\mathrm{Zr}_{0.5} \mathrm{Hf}_{0.5}\right)_{0.5} \mathrm{NiSn}_{0.998} \mathrm{Sb}_{0.002}$ & 1.5 \\
\hline $\mathrm{Hf}_{0.75} \mathrm{Zr}_{0.25} \mathrm{NiSn}_{0.99} \mathrm{Sb}_{0.01}$ & 1 \\
\hline $\mathrm{Hf}_{0.44} \mathrm{Zr}_{0.44} \mathrm{Ti}_{0.12} \mathrm{CoSb}_{0.8} \mathrm{Sn}_{0.2}$ & 1 \\
\hline
\end{tabular}

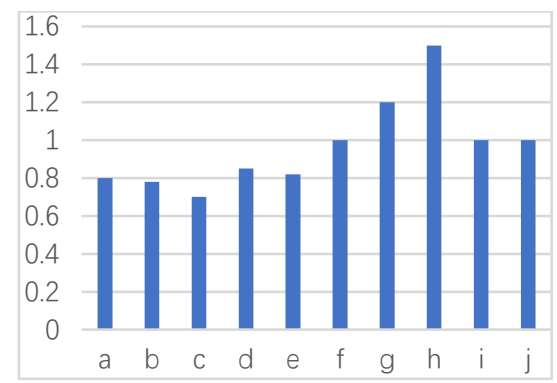

Figure 7. Maximum merit value of thermoelectricity of doped $\mathrm{HH}$

The above materials are usually used as traditional thermoelectric materials based on Seebeck effect. However it is hard for them to maintain good electrical conductivity and thermal insulation at the same time, that is, a compromise is required to be made among the three parameters of $\mathrm{S}, \sigma$ and $\kappa$. To avoid this problem and enhance the thermoelectric merit further, the anomalous Nernst effect (ANE) can be used. ANE, which means that a voltage perpendicular to the direction of heat flow and magnetization can be collected by applying a temperature gradient on magnetic materials, is also a kind of thermoelectric effects. The biggest difference between 
ANE and ordinary Nernst effect is that the former can increase rapidly even in a low magnetic field. ANE often occurs in Heusler compound, because the intrinsic ANE mainly depends on the Berry curvature near Fermi level rather than the magnetic moment itself [45],[46]. For the magnetic topological materials in Heusler compound, the Berry curvature induced by its band structure can produce additional anomalous transverse thermoelectric effect. Some experiments have shown that even if the total magnetization is zero, as long as the Berry curvature nearby is large enough, it can have very strong Nernst thermal efficiency [45]. The requirements of ANE for Berry curvature make it possible for many Heusler compounds to be good Nernst devices, such as Co2MnGa [45] verified by experiments, and $\mathrm{Ti} 2 \mathrm{MnX}(\mathrm{X}=\mathrm{Al}, \mathrm{Ga}, \mathrm{In})$ predicted in theory [46].

Nernst effect plays an important role in practical application. It can greatly reduce the use of p-type and ntype materials in devices. In the meantime, for transverse thermoelectric, when Nernst effect is applied to WSM, magnetic WSM is expected to generate topologically enhanced transverse thermoelectric merit value and realize thermoelectric energy conversion while its reverse process, the Etinghausen effect, can be used for solid-state refrigeration [47].

\subsection{Spintronics and Skyrmion}

With the development of topological quantum materials, topological spintronics has become a new favorite in the field of magnetism. Spin thermoelectronics studies the interaction between electron spin and thermal current. Based on the thermal spintronics, we have established many effective models. For example, the independent electronic effect in metals, such as the spin-dependent Seebeck, can be described by two parallel spin transport channels with different thermoelectric properties; in insulating ferromagnets, spin waves will cause collective effects like spin Seebeck. In general, the strong spin-orbital coupling can cause a series of anomalous physical properties, such as giant anomalous Hall effect and giant ANE.

In 2.1, we have seen how skyrmion causes the giant anomalous Hall effect. Skyrmion is a typical research object of thermal spintronics. It is a spin structure with quasi-particle properties and is protected by topology. Its two configurations are shown in Fig. 8 [48].
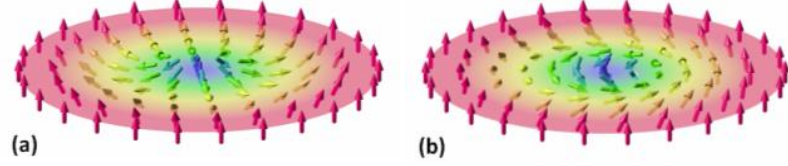

Figure 8. The vector field of two-dimensional magnetic skyrmions: (a) a hedgehog skyrmion and (b) a spiral skyrmion. [48]

It can be seen from the figure that the central spin of each skyrmion is oriented downward, which is antiparallel to the peripheral spin, while other spins rotate in the surface. This structure cannot be generated by the continuous change of ferromagnetic state. It is known that the skyrmion break the spatial inversion symmetry, so the magnetic materials without inversion symmetry, such as most of the semimetals, are good choices for the realization of the ground state of the skyrmion. In this kind of material, the anti-symmetric spin interaction - DzyaloshinskiiMoriya (DM) interaction [49] and Heisenberg interaction induce the ground state of the skyrmion. We have already observed stable skyrmion in the Heusler thin film alloy heterostructures $(\mathrm{Ta} / \mathrm{Co} 2 \mathrm{FeAl}(\mathrm{CFA}) / \mathrm{MgO})$ at room temperature [50].

\subsection{Topological Superconductivity}

Conventional superconductors can be transformed into topological superconductors by surface state modification. We have already introduced Fermi arc in 2.1. It is a unique surface state of WSM and when such topological state forms a superconducting state, the system has topological superconductivity. If a material is a topological superconductor, it will have a gap-free edge state. For the one-dimensional topological superconductor, this edge state is a zero-energy bound state at both ends of the system which is called Majorana fermion. For two-dimensional superconductors, the Majorana fermion is bound at the center of the vortex [51],[52]. The antiparticle of Majorana fermion is itself. Since the Majorana fermion has no charge, the quasi-particle made by the superposition of electrons and holes in superconducting system can be regarded as Majorana particles.

According to the symmetry of the superconducting gap, superconductors can be divided into s-wave superconductor, p-wave superconductor and d-wave superconductor. In normal s-wave superconductors, electrons with opposite spins are paired, but such quasiparticles are not equivalent to their antiparticles due to the existence of spin, that is, there is no Majorana fermion, so they are not topological superconductors. However, according to the theoretical prediction, if topological Weyl semimetal with time reversal symmetry forms $\mathrm{s}^{+-}$superconductivity (i.e., nonmagnetic Weyl semimetal), it will have topological superconductivity [53]. In addition, the superconducting states of fermions without spin also have topological superconductivity.

In the case of low temperature superconductivity, Cooper pairs appear. Cooper pair refers to the state in which electrons are bound together because when the energy of electrons is lower than Fermi energy, there is tiny gravity between electrons. The reason why it appears at low temperature is that at normal temperature, the energy of the thermal motion of electrons is very large, and the gravitational force between them is almost negligible. Experiments have already found that HF semimetals of YPtBi and LuPtBi have topological superconductivity [54],[55],[56], and their Cooper pairs not only have the total angular momentum of singlet and triplet states, but also have the states of quintet and septet with topological unusual points or line nodes [57]. Specifically, the topological surface states of LuPtBi and YPtBi originate from non-centrosymmetry of crystal structure caused by symmetry breaking [55] and the band inversion of energy band $\Gamma_{6}$ and $\Gamma_{8}$ [57]. Among them, the superconductivity on YPtBi surface state occurs below critical temperature $\mathrm{Tc}=0.77 \mathrm{~K}$, and the positive charge 
carrier density is extremely low at $\operatorname{Hc}(0)=1.5 \mathrm{~T}$, which is only $2 \times 10^{18} \mathrm{~cm}^{-3}$ [54], while the superconductivity of LuPtBi surface state occurs below $\mathrm{Tc}=1.0 \mathrm{~K}$ and $\mathrm{Hc}(0)=1.6$ $\mathrm{T}[55]$. In terms of element composition, some studies have found that [55] for XPtBi ( $\mathrm{X}$ is a rare earth element), when $\mathrm{X}$ is a light rare earth element, XPtBi tends to become a semiconductor, while when $\mathrm{X}$ is heavy, it tends to become a semimetal, and only when the orbital spin coupling interaction is involved, can it be a semimetal. Both $\mathrm{Lu}$ and $\mathrm{Y}$ are heavy rare earth elements with strong spin orbit coupling, so they can be excellent candidates for semimetals. In addition, HoPdBi, LnPd2Sn $(\mathrm{Ln}=\mathrm{Sc}, \mathrm{Y}$, $\mathrm{Lu})$ and other $\mathrm{HH}$ and $\mathrm{FH}$ also have topological superconductivity [58],[59].

\section{CONCLUSIONS AND PROSPECTS}

Heusler compounds can be divided into Full-Heusler, Inverse-Heusler and Half-Heusler according to different chemical structures; if take the shape of energy band as standard of classification, Heusler compound can be classified as conductor, insulator, topological insulator, semiconductor and semimetal; in the view of electronic structures, Heusler compounds can be ferromagnetic, paramagnetic or antiferromagnetic. The FH lattice can be regarded as the nesting of two zinc blende sublattices, so it has HgTe-like band structure. I-II-V type HH compounds, also known as Nowotny-Juza phase, are the most common HH. Under such elementary composition, if the total number of valence electrons is 18 , it will be a semiconductor material, otherwise, it is a metal or magnetic material. As for type I-III-IV HF, it does not contain rare earth and heavy metal elements, but shows strong band reversal.

In general, Heusler materials have good symmetry, which can protect topological phase in a better way. Heusler compounds have topological properties such as quantum anomalous Hall effect, topologically enhanced transverse Nernst thermoelectric effect and topological superconductivity. As far as thermoelectric properties are concerned, $\mathrm{HH}$ have higher Curie temperature and spin polarizability as well as more obvious thermoelectric properties than $\mathrm{FH}$.

Weyl semimetal will become a magnetic WSM with high spin polarizability in certain direction when the time reversal symmetry is broken and the central inversion symmetry is maintained. In $\mathrm{IH}$ and $\mathrm{FH}$, the inversion symmetry is broken naturally, so many Heusler compounds are WSM. In terms of physical properties, both topological insulator and WSM in Heusler materials have quantum anomalous Hall effect. The thermoelectric merit of Heusler compound can be improved by doping Heusler compound or introducing giant anomalous Nernst transverse thermoelectric effect. The above anomalous phenomena all come from the relatively high spin polarizability. In addition, the Heusler compounds also have the potential to become a topological superconductor.

It is hoped that we can explore more precise rules combined with specific elements and widely apply them to various devices.

\section{ACKNOWLEDGMENT}

I am indebted to my academic advisor Xiaoguang Li for his previous experience. My sincere thanks are due to Shanming Li for discussing the paper with me.

\section{REFERENCES}

1. T. Graf, C. Felser, and S. S. P. Parkin, "Simple rules for the understanding of Heusler compounds," Prog. Solid State Chem., vol. 39, no. 1, pp. 1-50, 2011.

2. K. Manna, Y. Sun, L. Muechler, J. Kübler, and C. Felser, "Heusler, Weyl and Berry," Nat. Rev. Mater., vol. 3, no. 8, pp. 244-256, 2018.

3. T. Graf, S. S. P. Parkin, and C. Felser, "Heusler compounds - A material class with exceptional properties," IEEE Trans. Magn., vol. 47, no. 2 PART 2, pp. 367-373, 2011.

4. M. S. Lee, F. P. Poudeu, and S. D. Mahanti, "Electronic structure and thermoelectric properties of Sb-based semiconducting half-Heusler compounds," Phys. Rev. B Condens. Matter Mater. Phys., vol. 83, no. 8, 2011.

5. P. Larson, S. D. Mahanti, S. Sportouch, and M. G. Kanatzidis, "Electronic structure of rare-earth nickel pnictides: Narrow-gap thermoelectric materials," Phys. Rev. B - Condens. Matter Mater. Phys., vol. 59, no. 24, pp. 15660-15668, 1999.

6. D. Xiao et al., "Half-Heusler compounds as a new class of three-dimensional topological insulators," Phys. Rev. Lett., vol. 105, no. 9, pp. 25-28, 2010.

7. P. Skomski, Ralph \& Komesu, Takashi \& Borca, Camelia \& Jeong, Hae \& Dowben, "Layer-resolved spin polarization in $\mathrm{Sb}$ overlayers on NiMnSb. Journal of Applied Physics." 2001.

8. I. Galanakis, K. Özdoğan, E. Şaşioğlu, and B. Aktaş, “Ab initio design of half-metallic fully compensated ferrimagnets: The case of $\mathrm{Cr}_{2} \mathrm{MnZ}(\mathrm{Z}=\mathrm{P}, \mathrm{As}, \mathrm{Sb}$, and $\mathrm{Bi})$," Phys. Rev. B - Condens. Matter Mater. Phys., vol. 75, no. 17, pp. 4-7, 2007.

9. S. Guha, S. Kumar, S. Datta, M. K. Manglam, and M. Kar, "Metal to semimetal transition and magnetic critical behavior at room temperature in the full Heusler alloy $\mathrm{Fe}_{2} \mathrm{MnSi}$," J. Phys. D. Appl. Phys., vol. 52, no. 50, p. 505002, 2019.

10. S. Öğ̈̈t and K. M. Rabe, "Band gap and stability in the ternary intermetallic compounds NiSnM (M=Ti,Zr,Hf): A first-principles study," Phys. Rev. B, vol. 51, no. 16, pp. 10443-10453, 1995.

11. G. H. Fecher, H. C. Kandpal, S. Wurmehl, C. Felser, and G. Schönhense, "Slater-Pauling rule and Curie temperature of Co2-based Heusler compounds," J. Appl. Phys., vol. 99, no. 8, pp. 2-4, 2006.

12. D. P. Oxley, R. S. Tebble, C. T. Slack, and K. C. Williams, "An anti-ferromagnetic heusler alloy, $\mathrm{Cu}_{2} \mathrm{MnSb}$," Nature, vol. 194, no. 4827. p. 465, 1962.

13. [13] R. Marrazza, D. Rossi and R. Ferro, "CaIn2-type and MgAgAs-type RESbPd compounds (RE=rare earth element)," Less Common Met., vol. 75, no. 2, p. 25, 1980.

14. S. K. Malik, A. M. Umarji, and G. K. Shenoy, "Magnetic and Mössbauer studies on rare-earth-containing Heusler alloys $\mathrm{Pd}_{2} \mathrm{RSn}(\mathrm{R}=\mathrm{Tb}-\mathrm{-} \mathrm{Yb})$," Phys. Rev. B, vol. 31, no. 11, pp. 6971-6975, 1985.

15. G. Andre, F. Bouree, A. Oles, W. Sikora, S. Baran, and A. Szytuła, "Magnetic structures of HoBdSb compound," Solid State Commun., vol. 104, no. 9, pp. 531-534, 1997.

16. D. Kaczorowski, K. Gofryk, T. Plackowski, A. LeitheJasper, and Y. Grin, "Unusual features of erbium-based 
Heusler phases,” J. Magn. Magn. Mater., vol. 290-291, pp. 573-579, 2005.

17. S. Ouardi, G. H. Fecher, C. Felser, and J. Kübler, "Realization of spin gapless semiconductors: The Heusler compound $\mathrm{Mn}_{2} \mathrm{CoAl}$," Phys. Rev. Lett., vol. 110, no. 10, pp. 2-6, 2013.

18. M. V Berry, "Quantal Phase Factors Accompanying Adiabatic Changes," Proc. R. Soc. Lond. A. Math. Phys. Sci., vol. 392, no. 1802, pp. 45-57, May 1984, [Online]. Available: http://www.jstor.org/stable/2397741.

19. S. Nie et al., "Magnetic Semimetals and Quantized Anomalous Hall Effect in EuB6," Phys. Rev. Lett., vol. 124, no. 7, pp. 76403, 2020.

20. YAO Shun-Yu DENG Ke ZHOU Shu-Yun†, "A brief introduction to type-II Weyl semimetals," Physics (College. Park. Md)., vol. 45, no. 10, pp. 635-639, 2016.

21. A. A. Soluyanov et al., "Type-II Weyl semimetals," Nature, vol. 527, no. 7579, pp. 495-498, 2015.

22. B. Yan and C. Felser, "Topological Materials: Weyl Semimetals," Annu. Rev. Condens. Matter Phys., vol. 8, no. 1, pp. 337-354, 2017.

23. L. Meng, J. Wu, J. Zhong, and R. A. Römer, “A type of robust superlattice type-I Weyl semimetal with four Weyl nodes," Nanoscale, vol. 11, no. 39, pp. 18358-18366, 2019.

24. S. Y. Xu et al., "Discovery of Lorentz-violating type II Weyl fermions in LaAlGe," Sci. Adv., vol. 3, no. 6, pp. 110, 2017.

25. G. Chang et al., "Room-temperature magnetic topological Weyl fermion and nodal line semimetal states in halfmetallic Heusler $\mathrm{Co}_{2} \mathrm{TiX}(\mathrm{X}=\mathrm{Si}$, Ge, or Sn)," Sci. Rep., vol. 6, no. November, pp. 1-9, 2016.

26. Y. W. Wei, C. K. Li, J. Qi, and J. Feng, "Magnetoconductivity of type-II Weyl semimetals," Phys. Rev. B, vol. 97, no. 20, pp. 1-9, 2018.

27. J. S. Bell and R. Jackiw, "A PCAC puzzle: $\pi_{0} \rightarrow \gamma \gamma$ in the $\sigma$ model,” Nuovo Cim. A, vol. 60, no. 1, pp. 47-61, 1969.

28. P. Hosur and X. Qi, "Recent developments in transport phenomena in Weyl semimetals," Comptes Rendus Phys., vol. 14, no. 9, pp. 857-870, 2013.

29. A. Lau, K. Koepernik, J. van den Brink, and C. Ortix, "Generic Coexistence of Fermi Arcs and Dirac Cones on the Surface of Time-Reversal Invariant Weyl Semimetals," Phys. Rev. Lett., vol. 119, no. 7, p. 76801, 2017.

30. Z. H. Liu et al., "Giant topological Hall effect in tetragonal Heusler alloy $\mathrm{Mn}_{2} \mathrm{PtSn}$," Scr. Mater., vol. 143, pp. 122 $125,2018$.

31. C. Shekhar et al., "Anomalous Hall effect in Weyl semimetal half-Heusler compounds RPtBi $(\mathrm{R}=\mathrm{Gd}$ and Nd)," Proc. Natl. Acad. Sci. U. S. A., vol. 115, no. 37, pp. 9140-9144, 2018.

32. A. A. Burkov, "Anomalous hall effect in weyl metals," Phys. Rev. Lett., vol. 113, no. 18, pp. 1-5, 2014

33. H. Hohl, A. P. Ramirez, C. Goldmann, G. Ernst, B. Wölfing, and E. Bucher, "Efficient dopants for ZrNiSnbased thermoelectric materials," J. Phys. Condens. Matter, vol. 11, no. 7, pp. 1697-1709, 1999.

34. Y. Xia, V. Ponnambalam, S. Bhattacharya, A. L. Pope, S. J. Poon, and T. M. Tritt, "Electrical transport properties of TiCoSb half-Heusler phases that exhibit high resistivity," J. Phys. Condens. Matter, vol. 13, no. 1, pp. 77-89, 2000.

35. S. R. Culp, S. J. Poon, N. Hickman, T. M. Tritt, and J. Blumm, "Effect of substitutions on the thermoelectric figure of merit of half-Heusler phases at $800^{\circ} \mathrm{C}$," Appl. Phys. Lett., vol. 88, no. 4, pp. 1-3, 2006.

36. S.-W. Kim, Y. Kimura, and Y. Mishima, "High temperature thermoelectric properties of TiNiSn-based half-Heusler compounds," Intermetallics, vol. 15, no. 3, pp. 349-356, 2007.
37. Q. Shen et al., "Effects of partial substitution of Ni by Pd on the thermoelectric properties of $\mathrm{ZrNiSn}$-based halfHeusler compounds," Appl. Phys. Lett., vol. 79, no. 25, pp. 4165-4167, 2001.

38. K. Gałązka, S. Populoh, W. Xie, S. Yoon, G. Saucke, and J. Hulliger, "Improved thermoelectric performance of $\left(\mathrm{Zr}_{0.3} \mathrm{Hf}_{0.7}\right) \mathrm{NiSn}$ half-Heusler compounds by $\mathrm{Ta}$ substitution," J. Appl. Phys., vol. 115, pp. 0-8, 2014.

39. O. Appel, M. Schwall, D. Mogilyansky, M. Köhne, B. Balke, and Y. Gelbstein, "Effects of Microstructural Evolution on the Thermoelectric Properties of SparkPlasma-Sintered $\quad \mathrm{Ti}_{0.3} \mathrm{Zr}_{0.35} \mathrm{Hf}_{0.35} \mathrm{NiSn}$ Half-Heusler Compound," J. Electron. Mater., vol. 42, no. 7, pp. 1340 1345, 2013

40. S. Populoh, M. H. Aguirre, O. C. Brunko, K. Galazka, Y. $\mathrm{Lu}$, and A. Weidenkaff, "High figure of merit in (Ti,Zr,Hf)NiSn half-Heusler alloys," Scr. Mater., vol. 66, no. 12, pp. 1073-1076, 2012.

41. M. Schwall and B. Balke, "Phase separation as a key to a thermoelectric high efficiency," Phys. Chem. Chem. Phys., vol. 15 , no. 6, pp. 1868-1872, 2013.

42. S. Sakurada and N. Shutoh, "Effect of Ti substitution on the thermoelectric properties of $(\mathrm{Zr}, \mathrm{Hf}) \mathrm{NiSn}$ half-Heusler compounds," Appl. Phys. Lett., vol. 86, no. 8, pp. 1-3, 2005.

43. G. Joshi, X. Yan, H. Wang, W. Liu, G. Chen, and Z. Ren, "Enhancement in Thermoelectric Figure-Of-Merit of an NType Half-Heusler Compound by the Nanocomposite Approach,” Adv. Energy Mater., vol. 1, pp. 643-647, 2011

44. X. Yan et al., "Thermoelectric property study of nanostructured p-type half-heuslers (Hf, Zr, Ti) $\mathrm{CoSb}_{0.8} \mathrm{Sn}_{0.2}$," Adv. Energy Mater., vol. 3, no. 9, pp. 1195-1200, 2013.

45. S. N. Guin et al., "Anomalous Nernst effect beyond the magnetization scaling relation in the ferromagnetic Heusler compound $\mathrm{Co}_{2} \mathrm{MnGa}$," NPG Asia Mater., vol. 11, no. 1,2019

46. J. Noky, J. Gayles, C. Felser, and Y. Sun, "Strong anomalous Nernst effect in collinear magnetic Weyl semimetals without net magnetic moments," Phys. Rev. B, vol. 97, no. 22, pp. 1-5, 2018.

47. A. E. De Paepe et al., "Zero-field Nernst effect in a ferromagnetic kagome-lattice Weyl-semimetal $\mathrm{Co}_{3} \mathrm{Sn}_{2} \mathrm{~S}_{2}$," J. Chem. Inf. Model., vol. 53, no. 9, pp. 1689-1699, 2019.

48. W. Contributors, "Magnetic skyrmion," Wikipedia, The Free Encyclopedia, 2020.

49. I. Dzyaloshinsky, "A thermodynamic theory of 'weak' ferromagnetism of antiferromagnetics," J. Phys. Chem. Solids, vol. 4, no. 4, pp. 241-255, 1958.

50. S. Husain et al., "Observation of Skyrmions at Room Temperature in $\mathrm{Co}_{2} \mathrm{FeAl}$ Heusler Alloy Ultrathin Film Heterostructures," Sci. Rep., vol. 9, no. 1, p. 1085, 2019.

51. F. Wilczek, "Majorana returns," Nat. Phys., vol. 5, no. 9, pp. 614-618, 2009.

52. N. Read and D. Green, "Paired states of fermions in two dimensions with breaking of parity and time-reversal symmetries and the fractional quantum Hall effect," Phys. Rev. B, vol. 61, no. 15, pp. 10267-10297, 2000.

53. P. Hosur, X. Dai, Z. Fang, and X.-L. Qi, "Time-reversalinvariant topological superconductivity in doped Weyl semimetals," Phys. Rev. B, vol. 90, no. 4, p. 45130, 2014.

54. N. P. Butch, P. Syers, K. Kirshenbaum, A. P. Hope, and J. Paglione, "Superconductivity in the topological semimetal YPtBi," vol. 220504, pp. 1-5, 2011.

55. F. F. Tafti, T. Fujii, S. Ren, and D. Cotret, "Superconductivity in the noncentrosymmetric halfHeusler compound LuPtBi : A candidate for topological superconductivity," vol. 184504, pp. 1-5, 2013. 
56. S. M. A. Radmanesh et al., "Evidence for unconventional superconductivity in half-Heusler YPdBi and TbPdBi compounds revealed by London penetration depth measurements," Phys. Rev. B, vol. 98, no. 24, p. 241111, 2018.

57. P. Brydon, L. Wang, M. Weinert, and D. Agterberg, "Pairing of $\mathrm{j}=3 / 2$ Fermions in Half-Heusler Superconductors," Phys. Rev. Lett., vol. 116, 2016.
58. P. Guo, J. Zhang, H. Yang, Z. Liu, K. Liu, and Z. Lu, " $\mathrm{LnPd}_{2} \mathrm{Sn}(\mathrm{Ln}=\mathrm{Sc}, \mathrm{Y}, \mathrm{Lu})$ class of Heusler alloys for topological superconductivity," pp. 1-5, 2018.

59. O. Pavlosiuk, D. Kaczorowski, X. Fabreges, A. Gukasov, and P. Wisniewski, "Antiferromagnetism and superconductivity in the half-Heusler semimetal HoPdBi," Sci. Rep., vol. 6, no. January, pp. 1-9, 2016. 